\title{
GERIATRIC UROLOGY
}

The importance of pelvic lymph node dissection in the elderly population: implications for interpreting the 2010 national comprehensive cancer network practice guidelines for bladder cancer treatment Abdollah F, Sun M, Shariat SF, Schmitges J, Djahangirian O, Tian Z, Jeldres C, Perrotte P, Montorsi F, Karakiewicz PI

Cancer Prognostics and Health Outcomes Unit, Montreal, Quebec, Canada; Department of Urology, Vita Salute San Raffaele University, Milan, Italy

J Urol. 2011; 185: 2078-84 
Purpose: National Comprehensive Cancer Network practice guidelines indicate that pelvic lymph node dissection can be omitted at radical cystectomy in elderly patients. We examined the pelvic lymph node dissection rate in patients 80 years old or older and the impact of pelvic lymph node dissection on cancer specific and overall mortality in these patients.

Materials and Methods: We examined the records of 11,183 patients treated with radical cystectomy in 17 Surveillance, Epidemiology and End Results registries. We performed univariate and multivariate Cox regression analysis to test the effect of pelvic lymph node dissection on cancer specific and overall mortality. Results: Overall pelvic lymph node dissection was omitted in $25 \%$ of patients, including $24.2 \%$ younger than 80 years and $30.8 \% 80$ years old or older $(\mathrm{p}<0.001)$. The 5 -year rate of freedom from cancer specific mortality for pelvic lymph node dissection vs no pelvic lymph node dissection was $62.5 \%$ vs $59.9 \%$ in patients younger than 80 years, and $50.0 \%$ vs $46.1 \%$ in those 80 years old or older $(\mathrm{p}=0.01$ and 0.005 , respectively). The 5-year rate of freedom from overall mortality for the same categories was $48.8 \%$ vs $43.9 \%$ and $28.3 \%$ vs $24.7 \%$ ( $p<0.001$ and 0.01 , respectively). On multivariate analysis omitting pelvic lymph node dissection was associated with a 1.3-fold higher cancer specific rate at ages less than 80 and 80 years or greater (each $\mathrm{p}<0.001$ ). Omitting pelvic lymph node dissection was also associated with a 1.3fold higher overall mortality rate, including 1.3 at ages less than 80 years and 1.2-fold at ages 80 years or greater (each $\mathrm{p} \leq 0.005)$.

Conclusions: Results indicate that pelvic lymph node dissection was more often omitted in patients 80 years old or older than in those younger than 80 years. However, the protective effect of pelvic lymph node dissection on cancer specific and overall mortality was virtually the same in the 2 age categories. Thus, advanced age should not be a limiting factor for performing pelvic lymph node dissection at radical cystectomy.

\section{Editorial Comment}

Unfortunately, evidence-based practice guidelines regarding the oncological management of elderly patients are sparse. In this scenario, authors are commended for highlighting that elderly patients might be denied the opportunity to receive the most effective treatment for their disease on the basis of chronological age alone.

Based on Surveillance, Epidemiology and End Results (SEER) database, pelvic lymph node dissection (PLND) was more often omitted in elderly patients ( $>80$ years) and when performed, tended to be less extensive ( $<10$ lymph nodes). However, metastasis rate was similar in patients younger than 80 and 80 years old or older $(26.1 \%$ vs $25.1 \%)$ and patients with a lymph node count of 10 or greater had significantly more favorable cancer specific and overall mortality rates than their counterparts with $<10$ lymph nodes. These results remained constant when patients were stratified by age less than 80 vs 80 years or greater.

Interestingly, PLND omission was less common in more contemporary study years, that is $38.6 \%$ between 1988 and 1992 vs $17.5 \%$ between 2003 and $2006(\mathrm{p}<0.001)$.

Although virtually all patients treated with radical cystectomy are judged to be fit candidates for such a major surgical procedure, future studies should consider comorbidities and the perspective of quality-of-life adjusted relative to survival benefits in a prospective design to confirm presented results, to provide better understanding of the effect of age on tumor biology and patient survival and to improve cancer care in the elderly.

Dr. Leonardo Oliveira Reis Assistant Professor of Urology University of Campinas, Unicamp Campinas, São Paulo, Brazil E-mail:reisleo@unicamp.br 